\title{
$\begin{array}{ll}\text { Research Square } & \begin{array}{l}\text { Preprints are preliminary reports that have not undergone peer review. } \\ \text { They should not be considered conclusive, used to inform clinical practice, } \\ \text { or referenced by the media as validated information. }\end{array}\end{array}$
}

\section{Clinically contextualised ECG interpretation}

\section{Charle André Viljoen ( $\sim$ charle.viljoen@uct.ac.za )}

University of Cape Town

\section{Rob Scott Millar}

University of Cape Town

\section{Kathryn Manning}

University of Cape Town

\section{Julian Hoevelmann}

Universitätsklinikum des Saarlandes, Saarland University Hospital

\section{Vanessa Celeste Burch}

University of Cape Town

\section{Research Article}

Keywords: electrocardiography, ECG, experiential learning, clinical context, case vignette

Posted Date: April 5th, 2021

DOI: https://doi.org/10.21203/rs.3.rs-362270/v1

License: (c) (i) This work is licensed under a Creative Commons Attribution 4.0 International License. Read Full License

Version of Record: A version of this preprint was published at BMC Medical Education on August 3rd, 2021. See the published version at https://doi.org/10.1186/s12909-021-02854-x. 


\section{Abstract}

Background ECGs are often taught without clinical context. However, in the clinical setting, ECGs are rarely interpreted without knowing the clinical presentation.

Methods We aimed to determine whether ECG diagnostic accuracy was influenced by knowledge of the clinical context and/or prior clinical exposure to the ECG diagnosis. Fourth- (junior) and sixth-year (senior) medical students as well as medical residents were invited to complete two multiple-choice question (MCQ) tests and a survey on the same day. Test 1 comprised 25 ECGs without case vignettes. Test 2, completed immediately after Test 1, comprised the same 25 ECGs and MCQs, but with case vignettes for each ECG. Subsequently, participants indicated in the survey when last, during prior clinical clerkships, they have seen each of the 25 conditions tested in Test 1 and 2.

Results This study comprised 176 participants, of which 67 (38.1\%) were junior students, 55 (31.3\%) were senior students and 54 (30.7\%) were medical residents. Prior ECG exposure depended on their level of training, i.e., junior students were exposed to $52 \%$ of the conditions tested, senior students $63.4 \%$ and residents $96.9 \%$. Overall, all groups showed a marginal improvement in accuracy of ECG interpretation when the clinical context was known to them (Cohen's $d=0.35, p<0.001$ ). The gains in accuracy were more pronounced amongst residents (Cohen's $d=0.59, p<$ 0.001 ), than amongst senior (Cohen's $d=0.38, p<0.001$ ) or junior students (Cohen's $d=0.29, p<0.001$ ). All participants were more likely to make a correct ECG diagnosis if they reported having seen the condition during prior clinical training, whether they were provided with a case vignette (odds ratio [OR] 1.46, 95\% confidence interval [CI] 1.24-1.71) or not (OR 1.58, 95\% Cl 1.35-1.84).

Conclusion ECG interpretation using clinical vignettes devoid of real patient experiences does not appear to have as great an impact on ECG diagnostic accuracy as prior exposure during clinical training. However, exposure to ECGs during clinical training is largely opportunistic and haphazard. ECG training should therefore not rely on experiential learning alone for teaching electrocardiography, but instead be supplemented by other formal methods of instruction.

\section{Introduction}

The electrocardiogram (ECG) is the most frequently used investigation to diagnose and monitor cardiac disease. ${ }^{1}$ Even after more than 120 years of use in clinical practice, there is no better investigation for the detection arrhythmias and conduction disturbances. ${ }^{2,3}$ Contemporary guidelines recommend an urgent ECG in any patient presenting with chest pain or suspected of having a myocardial ischaemia. ${ }^{4-6}$ Although the ECG is a powerful tool in diagnosing heart disease, incorrect interpretation of the ECG can lead to inappropriate clinical decisions with adverse outcomes. ${ }^{7}$

Over the past two decades, the lack of ECG competence has been well described for medical students, ${ }^{8-11}$ residents, $^{12-20}$ and qualified clinicians worldwide. ${ }^{21-26}$ Undergraduate $^{27-29}$ and postgraduate ECG curricula ${ }^{30-35}$ have been proposed in an attempt to standardise training in electrocardiography. However, to the best of our knowledge, exposure to the ECGs recommended by these curricular guidelines has not previously been reported for clinical clerkships or residency programmes. Furthermore, it is also unknown whether prior exposure to ECGs during clinical training has an impact on the accuracy of ECG interpretation.

ECG interpretation requires the recognition of visual patterns, ${ }^{36}$ which is a difficult skill to master. ${ }^{37}$ In other domains of Medicine, such as Dermatology and Radiology, where visual stimuli are also central to the diagnostic process, studies have shown that knowing the clinical context was associated with improved diagnostic accuracy. ${ }^{38,39}$ However, prior studies evaluating the impact of case vignettes on diagnostic accuracy in Electrocardiography per se

Page 2/19 
have yielded conflicting results. Grum et al. showed that knowing a clinical scenario did not influence the accuracy of ECG interpretation of third year medical students. ${ }^{40}$ However, third year students typically have little clinical experience compared to more senior trainees. On the contrary, Hatala et al. found that clinical scenarios were helpful in ECG interpretation. ${ }^{41,42}$ Knowing the clinical context was more useful in experienced ECG interpreters (i.e. residents) ${ }^{41,42}$ than novices (i.e. medical students) in particular. ${ }^{41}$ In this regard, arrhythmias remain under studied.

The aim of this study was therefore to determine whether diagnostic accuracy of ECG interpretation of medical students and residents was influenced by knowledge of the clinical context and/or prior clinical exposure to the condition.

\section{Methods}

We performed a cross-sectional study on undergraduate and postgraduate students from the University of Cape Town (UCT). The undergraduate students comprised fourth- and sixth-year medical students at the end of their Internal Medicine clerkship (enrolled in 2017). The postgraduate students were residents from the Department of Medicine (enrolled in 2018), with at least four years of working experience after graduating as medical doctors.

The study flow is outlined in Fig. 1. All participants completed two tests and a survey, on the same day, in the following order:

- During Test 1, participants were shown 25 ECGs. Each ECG was accompanied by a multiple-choice question (MCQ). For each question, there were five optional answers - four possible diagnoses (of which only one was correct), and a fifth option, i.e. "I am not sure what the answer is". Test 1 measured accuracy of ECG diagnoses in the absence of a case vignette.

- Immediately after submission of Test 1, participants completed Test 2. During Test 2, participants were shown the same 25 ECGs, which were now accompanied by a case vignette (Fig. 2). They answered the same MCQs and the same five optional answers as in Test 1. Test 2 accuracy of ECG diagnoses in the presence of a case vignette. The case vignettes described the patient demographic details (e.g., age and gender), common comorbidities associated with the condition (where appropriate) and typical clinical presentation to the emergency unit, ward or outpatient department, where the particular ECG was done.

- Once Test 2 was submitted, participants completed a survey in which they were asked to indicate when last during their prior clinical rotations they have seen the 25 ECGs that were included in Test 1 and Test 2. The survey measured exposure to ECGs during prior clinical training.

The invigilated, password protected, MCQ tests and survey were administered at the computer laboratories at the Faculty of Health Sciences. The 25 topics covered in the MCQ tests were considered to be core knowledge for undergraduate medical training at our institution. ${ }^{27}$ Of these, 12 were rhythm abnormalities (sinus arrhythmia, sinus arrest with escape rhythm, first degree AV block, Mobitz I and II second degree AV block, third degree AV block, atrial fibrillation [AF] with normal and uncontrolled rate, atrial flutter, AV node re-entrant tachycardia [AVNRT], ventricular tachycardia [VT] and ventricular fibrillation [VF]), and 13 were waveform abnormalities (left and right atrial enlargement, left ventricular hypertrophy [LVH], right ventricular hypertrophy [RVH], left bundle branch block [LBBB], right bundle branch block [RBBB], left anterior fascicular block, Wolff-Parkinson-White [WPW] pattern, anterior and 
inferior ST-segment elevation myocardial infarction [STEMI], pericarditis, hyperkalaemia, long QT syndrome). The ECGs and their answers are included in the Supplementary material.

The investigators of this study (three specialist physicians with a special interest in electrocardiography) agreed that the ECGs used in the tests were unequivocal examples of the conditions tested, and that the questions and multiplechoice options were fair for the given ECGs. Each correct answer was awarded one mark and negative marking was not applied. Participants were allowed 30 minutes to complete each test. The answers to the questions were only made available to the students after completion of both tests. The results of the MCQ tests in this study did not contribute to the participants' course marks.

\section{Statistical analysis}

Statistical analyses were performed on anonymised data using Stata (Version 14.2, StataCorp, College Station TX, USA). Descriptive statistics were used to summarise the ECG test scores and when last ECGs were seen during prior clinical training. For each ECG analysed, the proportion of correct answers for each cohort was calculated by the numerator/denominator and expressed as percentages. Within group change from Test 1 to Test 2 was analysed using McNemar's test (comparing diagnostic accuracy for each ECG in Test 1 and Test 2) and the signed-rank test (comparing total scores in Test 1 and Test 2). Cohen's $d$ was used to determine the effect size (practical significance) of the differences in total test scores, with values of $0.2,0.5$ and 0.8 indicating small, moderate and large effect sizes respectively. Associations between correct ECG diagnoses (with or without the provision of a case vignette) and whether the ECGs were seen during prior clinical training were assessed using odds ratios (OR) determined by logistic regression. Where applicable, a $p$ value of $<0.05$ was considered statistically significant and $95 \%$ confidence intervals (CI) were used to determine the precision of estimates.

\section{Results}

This study comprised 176 participants, of which 67 (38.1\%) were junior students, 55 (31.3\%) were senior students and 54 (30.7\%) were medical residents. All participants completed Test 1 and Test 2, as well as the survey.

\section{Exposure to ECGs during clinical training}

As illustrated in Fig. 3, junior medical students were exposed to just over half of the 25 ECGs in the tests by the end of their clinical clerkship, whereas senior medical students reported having seen about two thirds of these ECGs before graduating. Medical residents were exposed to almost all the ECGs during their clinical training. For all participants, most conditions were seen within 12 months prior to the study. Both junior and senior medical students were exposed to less arrhythmias during their clinical clerkships than ECGs with abnormal waveforms. AV blocks and ventricular arrhythmias were not frequently encountered by junior students (Mobitz type I second degree AV block [Wenckebach] 29.9\%, Mobitz type II second degree AV block 31.3\%, third degree AV block 28.4\%, ventricular tachycardia $47.8 \%$, ventricular fibrillation $32.8 \%$ ). Just more than half of the senior students were reportedly exposed to third degree AV block (56.4\%) and ventricular tachycardia (50.9\%) prior to graduation, but few reported prior exposure to Mobitz type I second degree AV block (Wenckebach, 45.5\%), Mobitz type II second degree AV block (36.4\%) or ventricular fibrillation (38.2\%). Few medical students were exposed to patients with AVNRT (junior 19.4\%, senior 20\%) or Wolff-ParkinsonWhite (junior $14.9 \%$, senior $21.8 \%$ ). Table 1 provides a detailed breakdown of which conditions each cohort had previously been exposed to during their clinical training. 
Table 1

ECGs reportedly seen during prior clinical training.

\begin{tabular}{|c|c|c|c|c|}
\hline & $\begin{array}{l}\text { Junior } \\
\text { students }\end{array}$ & $\begin{array}{l}\text { Senior } \\
\text { students }\end{array}$ & $\begin{array}{l}\text { Medical } \\
\text { residents }\end{array}$ & $\stackrel{p}{\text { value }}$ \\
\hline & $n=67$ & $n=55$ & $n=54$ & \\
\hline \multicolumn{5}{|l|}{ Arrhythmias } \\
\hline Sinus arrhythmia & $54(80.6)$ & $50(90.9)$ & $51(94.4)$ & 0.048 \\
\hline Sinus arrest & $13(19.4)$ & $19(34.5)$ & $49(90.7)$ & $<.001$ \\
\hline First degree AV block & $31(46.3)$ & $30(54.5)$ & $53(98.1)$ & $\hat{0} .001$ \\
\hline $\begin{array}{l}\text { Mobitz type I second degree AV block } \\
\text { (Wenckebach) }\end{array}$ & $20(29.9)$ & $25(45.5)$ & $53(98.1)$ & $\dot{<} 0.001$ \\
\hline Mobitz type II second degree AV block & $21(31.3)$ & $20(36.4)$ & $52(96.3)$ & $\stackrel{<}{0.001}$ \\
\hline Third degree AV block & $19(28.4)$ & $31(56.4)$ & $52(96.3)$ & $<.001$ \\
\hline Atrial fibrillation with normal rate & $57(85.1)$ & $47(85.5)$ & $54(100.0)$ & 0.012 \\
\hline Atrial fibrillation with uncontrolled rate & $42(62.7)$ & $47(85.5)$ & $54(100.0)$ & $\dot{<} 0.001$ \\
\hline Atrial flutter & $34(50.7)$ & $22(40.0)$ & $52(96.3)$ & $\dot{<} 0.001$ \\
\hline AVNRT & $13(19.4)$ & $11(20.0)$ & $52(96.3)$ & $\grave{0} .001$ \\
\hline Ventricular tachycardia & $32(47.8)$ & $28(50.9)$ & $53(98.1)$ & $\dot{<} .001$ \\
\hline Ventricular fibrillation & $22(32.8)$ & $21(38.2)$ & $53(98.1)$ & $\dot{<} 001$ \\
\hline \multicolumn{5}{|l|}{ Waveform abnormalities } \\
\hline Left anterior fascicular block & $21(31.3)$ & $21(38.2)$ & $49(90.7)$ & $\hat{0} .001$ \\
\hline Left bundle branch block & $45(67.2)$ & $50(90.9)$ & $54(100.0)$ & $\overleftarrow{0} .001$ \\
\hline Right bundle branch block & $38(56.7)$ & $45(81.8)$ & $52(96.3)$ & $\dot{<} .001$ \\
\hline Wolff-Parkinson-White pattern & $10(14.9)$ & $12(21.8)$ & $49(90.7)$ & $\overleftarrow{0} .001$ \\
\hline Left atrial enlargement & $45(67.2)$ & $48(87.3)$ & $52(96.3)$ & $\hat{<} .001$ \\
\hline Right atrial enlargement & $43(64.2)$ & $49(89.1)$ & $52(96.3)$ & $\hat{0}_{0.001}$ \\
\hline
\end{tabular}

Values are N(\%). 


\begin{tabular}{|lllll|}
\hline & $\begin{array}{l}\text { Junior } \\
\text { students }\end{array}$ & $\begin{array}{l}\text { Senior } \\
\text { students }\end{array}$ & $\begin{array}{l}\text { Medical } \\
\text { residents }\end{array}$ & $\begin{array}{l}p \\
\text { value }\end{array}$ \\
\hline Left ventricular hypertrophy & $63(94.0)$ & $55(100.0)$ & $54(100.0)$ & 0.036 \\
\hline Right ventricular hypertrophy & $47(70.1)$ & $49(89.1)$ & $53(98.1)$ & $<$ \\
Anterior STEMI & $56(83.6)$ & $54(98.2)$ & $54(100.0)$ & $<.001$ \\
\hline Inferior STEMI & $58(86.6)$ & $52(94.5)$ & $53(98.1)$ & 0.001 \\
\hline Pericarditis & $38(56.7)$ & $30(54.5)$ & $53(98.1)$ & $<$ \\
\hline Hyperkalaemia & $29(43.3)$ & $31(56.4)$ & $53(98.1)$ & $<$ \\
\hline Prolonged QT interval & $20(29.9)$ & $25(45.5)$ & $53(98.1)$ & $<$ \\
\hline Values are N (\%). & & & & 0.0001 \\
\hline
\end{tabular}

\section{Accuracy of ECG interpretation with or without a case vignette}

Figure 4A demonstrates the proportion of ECGs that were correctly analysed when participants were provided with a case vignette or not. Overall, all groups showed a marginal improvement in accuracy of ECG interpretation when the clinical context was known to them (Cohen's $d=0.35, p<0.001$ ). The gains in accuracy were more pronounced amongst the residents (Cohen's $d=0.59, p<0.001$ ), than amongst senior (Cohen's $d=0.38, p<0.001$ ) or junior students (Cohen's $d=0.29, p<0.001$ ).

As shown in Fig. 4B, subgroup analyses showed that junior students were not influenced by case vignettes when interpreting arrhythmias. Although statistically significant, senior students and residents showed an increase of only 3 percentage units in their scores of the arrhythmia section of the tests, when they were provided with case vignettes. All groups showed an increase in diagnostic accuracy when they were provided with a case vignette for ECGs with abnormal waveforms (Fig. 4C). Table 2 provides a detailed breakdown of how each cohort's ECG diagnostic accuracy was influenced by whether they were provided with a case vignette or not at the time of ECG analysis. 
Table 2

Proportion of ECGs correctly diagnosed when participants were provided with a case vignette or not.

$\begin{array}{lll}\text { Junior students } & \text { Senior students } & \text { Medical } \\ n=67 & n=55 & n=54\end{array}$

\begin{tabular}{|c|c|c|c|c|c|c|c|}
\hline & $\begin{array}{l}\text { With } \\
\text { scenario }\end{array}$ & $\begin{array}{l}\text { p- } \\
\text { value }\end{array}$ & $\begin{array}{l}\text { Wit } \\
\text { sce }\end{array}$ & $\begin{array}{l}\text { With } \\
\text { scenario }\end{array}$ & $\begin{array}{l}\text { P- } \\
\text { value }\end{array}$ & & 1 \\
\hline
\end{tabular}

\section{Arrhythmias}

\begin{tabular}{|c|c|c|c|c|c|c|c|c|c|}
\hline $\begin{array}{l}\text { Sinus } \\
\text { arrhythmia }\end{array}$ & $\begin{array}{l}59 \\
(88.1)\end{array}$ & $\begin{array}{l}59 \\
(88.1)\end{array}$ & 1 & $\begin{array}{l}41 \\
(74.5)\end{array}$ & $\begin{array}{l}47 \\
(85.5)\end{array}$ & 0.083 & $\begin{array}{l}51 \\
(94.4)\end{array}$ & $\begin{array}{l}49 \\
(90.7)\end{array}$ & 0.317 \\
\hline Sinus arrest & $\begin{array}{l}55 \\
(82.1)\end{array}$ & $\begin{array}{l}56 \\
(83.6)\end{array}$ & 0.706 & $\begin{array}{l}39 \\
(70.9)\end{array}$ & $\begin{array}{l}44 \\
(80.0)\end{array}$ & 0.096 & $\begin{array}{l}43 \\
(79.6)\end{array}$ & $\begin{array}{l}43 \\
(79.6)\end{array}$ & 1 \\
\hline $\begin{array}{l}\text { First degree AV } \\
\text { block }\end{array}$ & $\begin{array}{l}54 \\
(80.6)\end{array}$ & $\begin{array}{l}49 \\
(73.1)\end{array}$ & 0.059 & $\begin{array}{l}38 \\
(69.1)\end{array}$ & $\begin{array}{l}40 \\
(72.7)\end{array}$ & 0.527 & $\begin{array}{l}47 \\
(87.0)\end{array}$ & $\begin{array}{l}51 \\
(94.4)\end{array}$ & 0.103 \\
\hline $\begin{array}{l}\text { Mobitz type I } \\
\text { second degree } \\
\text { AV block }\end{array}$ & $\begin{array}{l}49 \\
(73.1)\end{array}$ & $\begin{array}{l}49 \\
(73.1)\end{array}$ & 1 & $\begin{array}{l}30 \\
(54.5)\end{array}$ & $\begin{array}{l}29 \\
(52.7)\end{array}$ & 0.781 & $\begin{array}{l}32 \\
(59.3)\end{array}$ & $\begin{array}{l}29 \\
(53.7)\end{array}$ & 0.317 \\
\hline $\begin{array}{l}\text { Mobitz type II } \\
\text { second degree } \\
\text { AV block }\end{array}$ & $\begin{array}{l}54 \\
(80.6)\end{array}$ & $\begin{array}{l}52 \\
(77.6)\end{array}$ & 0.480 & $\begin{array}{l}33 \\
(60.0)\end{array}$ & $\begin{array}{l}37 \\
(67.3)\end{array}$ & 0.317 & $\begin{array}{l}45 \\
(83.3)\end{array}$ & $\begin{array}{l}40 \\
(74.1)\end{array}$ & 0.059 \\
\hline $\begin{array}{l}\text { Third degree } \\
\text { AV block }\end{array}$ & $\begin{array}{l}57 \\
(85.1)\end{array}$ & $\begin{array}{l}54 \\
(80.6)\end{array}$ & 0.257 & $\begin{array}{l}38 \\
(69.1)\end{array}$ & $\begin{array}{l}39 \\
(70.9)\end{array}$ & 0.563 & $\begin{array}{l}51 \\
(94.4)\end{array}$ & $\begin{array}{l}50 \\
(92.6)\end{array}$ & 0.654 \\
\hline $\begin{array}{l}\text { Atrial } \\
\text { fibrillation with } \\
\text { normal rate }\end{array}$ & $\begin{array}{l}56 \\
(83.6)\end{array}$ & $\begin{array}{l}58 \\
(86.6)\end{array}$ & 0.414 & $\begin{array}{l}36 \\
(65.5)\end{array}$ & $\begin{array}{l}40 \\
(72.7)\end{array}$ & 0.103 & $\begin{array}{l}47 \\
(87.0)\end{array}$ & $\begin{array}{l}51 \\
(94.4)\end{array}$ & 0.103 \\
\hline $\begin{array}{l}\text { Atrial } \\
\text { fibrillation with } \\
\text { uncontrolled } \\
\text { rate }\end{array}$ & $\begin{array}{l}49 \\
(73.1)\end{array}$ & $\begin{array}{l}45 \\
(67.2)\end{array}$ & 0.248 & $\begin{array}{l}33 \\
(60.0)\end{array}$ & $\begin{array}{l}28 \\
(50.9)\end{array}$ & 0.197 & $\begin{array}{l}30 \\
(55.6)\end{array}$ & $\begin{array}{l}41 \\
(75.9)\end{array}$ & 0.002 \\
\hline Atrial flutter & $\begin{array}{l}43 \\
(64.2)\end{array}$ & $\begin{array}{l}41 \\
(61.2)\end{array}$ & 0.593 & $\begin{array}{l}30 \\
(54.5)\end{array}$ & $\begin{array}{l}35 \\
(63.6)\end{array}$ & 0.197 & $\begin{array}{l}36 \\
(66.7)\end{array}$ & $\begin{array}{l}36 \\
(66.7)\end{array}$ & 1 \\
\hline $\begin{array}{l}\text { AV nodal re- } \\
\text { entrant } \\
\text { tachycardia }\end{array}$ & $\begin{array}{l}40 \\
(59.7)\end{array}$ & $\begin{array}{l}50 \\
(74.6)\end{array}$ & 0.012 & $\begin{array}{l}27 \\
(49.1)\end{array}$ & $\begin{array}{l}32 \\
(58.2)\end{array}$ & 0.096 & $\begin{array}{l}44 \\
(81.5)\end{array}$ & $\begin{array}{l}49 \\
(90.7)\end{array}$ & 0.059 \\
\hline $\begin{array}{l}\text { Ventricular } \\
\text { tachycardia }\end{array}$ & $\begin{array}{l}39 \\
(58.2)\end{array}$ & $\begin{array}{l}42 \\
(62.7)\end{array}$ & 0.257 & $\begin{array}{l}34 \\
(61.8)\end{array}$ & $\begin{array}{l}33 \\
(60.0)\end{array}$ & 0.739 & $\begin{array}{l}42 \\
(77.8)\end{array}$ & $\begin{array}{l}47 \\
(87.0)\end{array}$ & 0.132 \\
\hline $\begin{array}{l}\text { Ventricular } \\
\text { fibrillation }\end{array}$ & $\begin{array}{l}30 \\
(44.8)\end{array}$ & $\begin{array}{l}36 \\
(53.7)\end{array}$ & 0.109 & $\begin{array}{l}27 \\
(49.1)\end{array}$ & $\begin{array}{l}24 \\
(43.6)\end{array}$ & 0.257 & $\begin{array}{l}33 \\
(61.1)\end{array}$ & $\begin{array}{l}36 \\
(66.7)\end{array}$ & 0.257 \\
\hline \multicolumn{10}{|c|}{ Waveform abnormalities } \\
\hline $\begin{array}{l}\text { Left anterior } \\
\text { fascicular } \\
\text { block }\end{array}$ & $\begin{array}{l}50 \\
(74.6)\end{array}$ & $\begin{array}{l}49 \\
(73.1)\end{array}$ & 0.808 & $\begin{array}{l}29 \\
(52.7)\end{array}$ & $\begin{array}{l}24 \\
(43.6)\end{array}$ & 0.166 & $\begin{array}{l}39 \\
(72.2)\end{array}$ & $\begin{array}{l}42 \\
(77.8)\end{array}$ & 0.257 \\
\hline $\begin{array}{l}\text { Left bundle } \\
\text { branch block }\end{array}$ & $\begin{array}{l}58 \\
(86.6)\end{array}$ & $\begin{array}{l}56 \\
(83.6)\end{array}$ & 0.317 & $\begin{array}{l}38 \\
(69.1)\end{array}$ & $\begin{array}{l}38 \\
(69.1)\end{array}$ & 1 & $\begin{array}{l}50 \\
(92.6)\end{array}$ & $\begin{array}{l}49 \\
(90.7)\end{array}$ & 0.564 \\
\hline
\end{tabular}




\begin{tabular}{|c|c|c|c|c|c|c|c|c|c|}
\hline & \multicolumn{3}{|c|}{ Junior students } & \multicolumn{3}{|c|}{ Senior students } & \multicolumn{3}{|c|}{ Medical residents } \\
\hline & \multicolumn{3}{|l|}{$n=67$} & \multicolumn{3}{|l|}{$n=55$} & \multicolumn{3}{|l|}{$n=54$} \\
\hline & $\begin{array}{l}\text { Without } \\
\text { scenario }\end{array}$ & $\begin{array}{l}\text { With } \\
\text { scenario }\end{array}$ & $\begin{array}{l}\mathrm{p}- \\
\text { value }\end{array}$ & $\begin{array}{l}\text { Without } \\
\text { scenario }\end{array}$ & $\begin{array}{l}\text { With } \\
\text { scenario }\end{array}$ & $\begin{array}{l}\mathrm{p}- \\
\text { value }\end{array}$ & $\begin{array}{l}\text { Without } \\
\text { scenario }\end{array}$ & $\begin{array}{l}\text { With } \\
\text { scenario }\end{array}$ & $\begin{array}{l}\mathrm{p}- \\
\text { value }\end{array}$ \\
\hline $\begin{array}{l}\text { Right bundle } \\
\text { branch block }\end{array}$ & $\begin{array}{l}55 \\
(82.1)\end{array}$ & $\begin{array}{l}55 \\
(82.1)\end{array}$ & 1 & $\begin{array}{l}42 \\
(76.4)\end{array}$ & $\begin{array}{l}42 \\
(76.4)\end{array}$ & 1 & $\begin{array}{l}48 \\
(88.9)\end{array}$ & $\begin{array}{l}49 \\
(90.7)\end{array}$ & 0.655 \\
\hline $\begin{array}{l}\text { Wolff- } \\
\text { Parkinson- } \\
\text { White pattern }\end{array}$ & $\begin{array}{l}49 \\
(73.1)\end{array}$ & $\begin{array}{l}59 \\
(88.1)\end{array}$ & 0.012 & $\begin{array}{l}37 \\
(67.3)\end{array}$ & $\begin{array}{l}47 \\
(85.5)\end{array}$ & 0.004 & $\begin{array}{l}47 \\
(87.0)\end{array}$ & $\begin{array}{l}52 \\
(96.3)\end{array}$ & 0.059 \\
\hline $\begin{array}{l}\text { Left atrial } \\
\text { enlargement }\end{array}$ & $\begin{array}{l}52 \\
(77.6)\end{array}$ & $\begin{array}{l}51 \\
(76.1)\end{array}$ & 0.763 & $\begin{array}{l}38 \\
(69.1)\end{array}$ & $\begin{array}{l}41 \\
(74.5)\end{array}$ & 0.439 & $\begin{array}{l}46 \\
(85.2)\end{array}$ & $\begin{array}{l}45 \\
(83.3)\end{array}$ & 0.739 \\
\hline $\begin{array}{l}\text { Right atrial } \\
\text { enlargement }\end{array}$ & $\begin{array}{l}54 \\
(80.6)\end{array}$ & $\begin{array}{l}57 \\
(85.1)\end{array}$ & 0.179 & $\begin{array}{l}43 \\
(78.2)\end{array}$ & $\begin{array}{l}45 \\
(81.8)\end{array}$ & 0.527 & $\begin{array}{l}47 \\
(87.0)\end{array}$ & $\begin{array}{l}49 \\
(90.7)\end{array}$ & 0.414 \\
\hline $\begin{array}{l}\text { Left ventricular } \\
\text { hypertrophy }\end{array}$ & $\begin{array}{l}46 \\
(68.7)\end{array}$ & $\begin{array}{l}58 \\
(86.6)\end{array}$ & 0.003 & $\begin{array}{l}44 \\
(80.0)\end{array}$ & $\begin{array}{l}49 \\
(89.1)\end{array}$ & 0.059 & $\begin{array}{l}49 \\
(90.7)\end{array}$ & $\begin{array}{l}51 \\
(94.4)\end{array}$ & 0.414 \\
\hline $\begin{array}{l}\text { Right } \\
\text { ventricular } \\
\text { hypertrophy }\end{array}$ & $\begin{array}{l}47 \\
(70.1)\end{array}$ & $\begin{array}{l}63 \\
(94.0)\end{array}$ & $\begin{array}{l}< \\
0.001\end{array}$ & $\begin{array}{l}31 \\
(56.4)\end{array}$ & $\begin{array}{l}48 \\
(87.3)\end{array}$ & $<001$ & $\begin{array}{l}44 \\
(81.5)\end{array}$ & $\begin{array}{l}51 \\
(94.4)\end{array}$ & 0.020 \\
\hline $\begin{array}{l}\text { Anterior ST- } \\
\text { segment } \\
\text { elevation } \\
\text { myocardial } \\
\text { infarction }\end{array}$ & $\begin{array}{l}62 \\
(92.5)\end{array}$ & $\begin{array}{l}63 \\
(94.0)\end{array}$ & 0.564 & $\begin{array}{l}46 \\
(83.6)\end{array}$ & $\begin{array}{l}48 \\
(87.3)\end{array}$ & 0.480 & $\begin{array}{l}52 \\
(96.3)\end{array}$ & $\begin{array}{l}51 \\
(94.4)\end{array}$ & 0.564 \\
\hline $\begin{array}{l}\text { Inferior ST- } \\
\text { segment } \\
\text { elevation } \\
\text { myocardial } \\
\text { infarction }\end{array}$ & $\begin{array}{l}61 \\
(91.0)\end{array}$ & $\begin{array}{l}58 \\
(86.6)\end{array}$ & 0.180 & $\begin{array}{l}49 \\
(89.1)\end{array}$ & $\begin{array}{l}48 \\
(87.3)\end{array}$ & 0.564 & $\begin{array}{l}44 \\
(81.5)\end{array}$ & $\begin{array}{l}48 \\
(88.9)\end{array}$ & 0.046 \\
\hline Pericarditis & $\begin{array}{l}30 \\
(44.8)\end{array}$ & $\begin{array}{l}57 \\
(85.1)\end{array}$ & $\begin{array}{l}<.001 \\
0.00\end{array}$ & $\begin{array}{l}20 \\
(36.4)\end{array}$ & $\begin{array}{l}40 \\
(72.7)\end{array}$ & $<.001$ & $\begin{array}{l}37 \\
(68.5)\end{array}$ & $\begin{array}{l}51 \\
(94.4)\end{array}$ & 0.001 \\
\hline Hyperkalaemia & $\begin{array}{l}39 \\
(58.2)\end{array}$ & $\begin{array}{l}55 \\
(82.1)\end{array}$ & $\begin{array}{l}< \\
0.001\end{array}$ & $\begin{array}{l}18 \\
(32.7)\end{array}$ & $\begin{array}{l}34 \\
(61.8)\end{array}$ & $\begin{array}{l}< \\
0.001\end{array}$ & $\begin{array}{l}29 \\
(53.7)\end{array}$ & $\begin{array}{l}45 \\
(83.3)\end{array}$ & $\begin{array}{l}< \\
0.001\end{array}$ \\
\hline $\begin{array}{l}\text { Prolonged QT } \\
\text { interval }\end{array}$ & $\begin{array}{l}54 \\
(80.6)\end{array}$ & $\begin{array}{l}57 \\
(85.1)\end{array}$ & 0.405 & $\begin{array}{l}38 \\
(69.1)\end{array}$ & $\begin{array}{l}43 \\
(78.2)\end{array}$ & 0.132 & $\begin{array}{l}43 \\
(79.6)\end{array}$ & $\begin{array}{l}48 \\
(88.9)\end{array}$ & 0.096 \\
\hline
\end{tabular}

\section{Impact of prior real life ECG exposure on diagnostic accuracy}

All participants were more likely to make a correct ECG diagnosis if they reported having seen the condition during prior clinical training (Fig. 5). This was true for all groups, whether they were provided with a case vignette (OR 1.46, $95 \% \mathrm{Cl} 1.24-1.71$ ) or not (OR 1.58, 95\% Cl 1.35-1.84). The largest impact was amongst residents, who were almost six times more likely to make a correct diagnosis when they were given an ECG of a condition that they reported to have seen during prior clinical training. 
In this study, we set out to determine whether clinically contextualised ECG interpretation, using case vignettes, improved medical students and residents' ECG diagnostic accuracy. We found that trainees benefited only marginally from these case vignettes in improving their ECG diagnostic accuracy. However, students and residents were more likely to make the correct ECG diagnosis if they had previously seen the condition during prior clinical training. In light of this finding, it was of concern that, during clinical clerkships, trainees were not exposed to all the ECGs they were expected to be able to interpret by graduation.

In the classroom, ECGs are often taught without clinical context. However, in real life, ECGs are performed in settings where the ECG interpreter is likely to know the clinical presentation. Indeed, we found that students and residents were more accurate at diagnosing $\mathrm{RVH}$, pericarditis and hyperkalaemia, when they knew the clinical context. Of course, in these cases, it is possible that the participant could predict the correct answer from the history alone. ${ }^{42,43}$ However, these ECG diagnoses can be very challenging without knowing the clinical context, especially for the novice ECG interpreter. Knowing the demographic details and risk factor profile of a patient will help to make the differentiation between pericarditis and myocardial infarction, as opposed to only looking at the ECGs. Similarly, the clinical context is important in the analysis of bradycardias, as hyperkalaemia can easily be mistaken for third degree AV block on an ECG. The distinction is of paramount importance, as the management is very different.

However, one should not consider the impact of case vignettes on the interpretation of single ECG diagnoses. In this study, we tested the impact of case vignettes on the interpretation of ECGs on a wide array of arrhythmias and waveform abnormalities. Students and residents were only slightly more accurate in their ECG interpretation when provided with a case vignette. We found that the largest value in improved diagnostic accuracy was for ECGs showing abnormal waveforms, as has been described by Hatala et al. ${ }^{41}$ Our study further supports their findings, in that medical trainees with different levels of expertise all benefited from being provided with a clinical scenario when interpreting ECGs with abnormal waveforms. Our study also supports the study by Grum et al., that showed that clinical scenarios had no significant impact on junior medical students' interpretation of atrial fibrillation and supraventricular tachycardia. ${ }^{40}$ In addition to the latter, our study also included AV blocks, atrial flutter and ventricular tachycardia. We found that junior medical students (with little clinical experience) did not benefit from case vignettes when interpreting these arrhythmias. This could potentially be explained by their lack of exposure to patients presenting with rhythm disturbances during clinical clerkships in general medical wards. This is a cause for major concern, as there is little formal ECG training after graduation. ${ }^{27}$

Medical training has traditionally relied on experiential learning, i.e., the acquisition of knowledge and skills from clinical exposure. ${ }^{44}$ Indeed, there is a close relationship between the accrual of clinical experience and increased competence. ${ }^{45}$ As has been shown for the interpretation of chest radiographs, ${ }^{46,47}$ we found that ECG diagnostic accuracy increased with more advanced levels of training. Our study results were also consistent with the literature reporting that diagnostic accuracy is positively influenced by prior exposure to examples of similar conditions. ${ }^{48}$ Moreover, increased exposure and repeated practice are known to be associated with better ECG diagnostic accuracy. ${ }^{49,50}$ We therefore propose that ECG exposure should be maximised during clinical clerkships, to ensure that undergraduate and postgraduate trainees become familiar with the conditions specified and recommended by undergraduate ${ }^{27-29}$ and postgraduate ECG curricula. ${ }^{30-35}$

Merely being present on ward rounds or in the clinic or emergency unit, does not result in the acquisition of ECG competence. Instead, students and residents should be actively encouraged to analyse and interpret ECGs during their clinical training. This largely self-directed learning pursuit may assist in gaining more experience, as well as contextualising ECG learning. ${ }^{51}$ ECG learning in the clinical setting should be supported by using mobile learning 
strategies, ${ }^{52}$ which may further enhance contextualised learning. ${ }^{53,54}$ In this regard, there is evidence that the use of algorithm-based ECG reference apps may be of greater benefit than unguided exploration of the Internet. ${ }^{55}$ However, there is limited exposure during clinical clerkships to conditions that medical students are expected to diagnose. ${ }^{27}$ This implies that ECG training should not rely on experiential learning alone for teaching electrocardiography. Instead, ECG training should be supplemented by other formal methods of instruction. ${ }^{56-58}$

\section{Study limitations}

The lack of exposure to the clinical conditions tested in this study pertains to students trained at our institution. These results would need to be validated by follow up studies at more centres, around the world. However, it does point out that, unless a medical student spends dedicated time in clinical clerkships such as Cardiology or Emergency Medicine during their undergraduate training, they might not encounter these conditions.

Our study only tested the impact of case vignettes that were appropriate to the ECGs that were provided. We did not include misleading clinical scenarios, to evaluate if these would impede diagnostic accuracy. Although only tested on limited ECGs, it has been shown before that misleading case vignettes are detrimental to ECG diagnostic accuracy. ${ }^{41}$

\section{Conclusion}

The main messages of this study are that exposure to patients with ECG abnormalities during clinical clerkships is key to learning interpret ECGs. ECG interpretation using case vignettes devoid of real patient experiences does not have the same impact on ECG diagnostic accuracy. However, exposure to ECGs during clinical training is largely opportunistic and haphazard. The mandate to supplement clerkship-based ECG training using structured learning activities, including mobile learning, is clear. The evidence to support these learning strategies is emerging in the literature.

\section{Declarations}

\section{Ethical approval and consent to participate}

Ethical approval was obtained from the Human Research Ethics Committee (HREC) at the University of Cape Town's Faculty of Health Sciences (HREC reference number 046/2016 for undergraduate students and 080/2018 for postgraduate students) as well as institutional permission from the Department of Student Affairs at the University of Cape Town. All participants signed informed consent prior to enrolment in the study. All methods were carried out in accordance with relevant guidelines and regulations.

\section{Consent for publication}

All study participants provided informed consent for the anonymised analysis of their responses in the three rounds of the modified Delphi study and a possible scientific publication of the results.

\section{Availability of data and materials}

The datasets used and/or analysed during the current study, are available in the "Clinically contextualised ECG interpretation" repository, which could be accessed at https://doi.org/10.25375/uct.14297708.v1.

The ECGs contained in the supplementary material are the property of the University of Cape Town, and when used, these should be referenced as such. 


\section{Competing interests}

The authors report no conflicts of interest. The authors alone are responsible for the content and writing of the article.

\section{Funding}

RSM is a lecturer and host of the AO Memorial Advanced ECG and Arrhythmia Course and receives an honorarium from Medtronic Africa. The authors did not receive a specific grant for this research from any funding agency in the public, commercial or not-for-profit sectors.

\section{Acknowledgements}

The authors wish to thank Ms Elani Muller and Ms Sylvia Dennis and from the Hatter Institute for Cardiovascular Research in Africa (HICRA) for their very valuable input and assistance. We are grateful to the academic staff, Professor Nicci Wearne, Dr Ayanada Gcelu, Ms Sipho Mankayi, Ms Zanele Magwa and Ms Janine Daniels, for their assistance with the lectures and invigilating at the ECG assessments. Finally, we would like to thank our students and residents for their dedication in taking part in the study and their very valuable feedback to help improve undergraduate and postgraduate ECG training.

\section{Authors' contributions}

CAV conceived the study protocol, with advice from RSM and VCB regarding study design. CAV collected the data. CAV performed the statistical analysis under the guidance of $\mathrm{JH}$ and KM. CAV, RSM, JH and VCB interpreted the results. CV drafted the manuscript, which was critically revised by RSM, KM, JH and VCB. All authors have read and approved the manuscript.

\section{References}

1. Kashou A, May A, DeSimone C, Noseworthy P. The essential skill of ECG interpretation: How do we define and improve competency? Postgrad Med J. 2020;96(1133):125-7.

2. Mason JW, Hancock EW, Gettes LS, American Heart Association E, Arrhythmias Committee CoCC, American College of Cardiology $\mathrm{F}$, et al. Recommendations for the standardization and interpretation of the electrocardiogram: part II: Electrocardiography diagnostic statement list: a scientific statement from the American Heart Association Electrocardiography and Arrhythmias Committee, Council on Clinical Cardiology; the American College of Cardiology Foundation; and the Heart Rhythm Society: endorsed by the International Society for Computerized Electrocardiology. Circulation. 2007;115(10):1325-32.

3. Hindricks G, Potpara T, Dagres N, Arbelo E, Bax JJ, Blomstrom-Lundqvist C, et al. 2020 ESC Guidelines for the diagnosis and management of atrial fibrillation developed in collaboration with the European Association of Cardio-Thoracic Surgery (EACTS). Eur Heart J. 2020.

4. Ibanez B, James S, Agewall S, Antunes MJ, Bucciarelli-Ducci C, Bueno H, et al. 2017 ESC Guidelines for the management of acute myocardial infarction in patients presenting with ST-segment elevation: The Task Force for the management of acute myocardial infarction in patients presenting with ST-segment elevation of the European Society of Cardiology (ESC). Eur Heart J. 2018;39(2):119-77.

5. Collet JP, Thiele H, Barbato E, Barthelemy O, Bauersachs J, Bhatt DL, et al. 2020 ESC Guidelines for the management of acute coronary syndromes in patients presenting without persistent ST-segment elevation. Eur Heart J. 2020. 
6. Knuuti J, Wijns W, Saraste A, Capodanno D, Barbato E, Funck-Brentano C, et al. 2019 ESC Guidelines for the diagnosis and management of chronic coronary syndromesThe Task Force for the diagnosis and management of chronic coronary syndromes of the European Society of Cardiology (ESC). European Heart Journal. 2019.

7. Mele P. Improving electrocardiogram interpretation in the clinical setting. Journal of electrocardiology. 2008;41(5):438-9.

8. Jablonover RS, Lundberg E, Zhang Y, Stagnaro-Green A. Competency in electrocardiogram interpretation among graduating medical students. Teach Learn Med. 2014;26(3):279-84.

9. Kopec G, Magon W, Holda M, Podolec P. Competency in ECG interpretation among medical students. Med Sci Monitor. 2015;21.

10. Lever NA, Larsen PD, Dawes M, Wong A, Harding SA. Are our medical graduates in New Zealand safe and accurate in ECG interpretation? The New Zealand medical journal. 2009;122(1292):9-15.

11. Little B, Mainie I, Ho KJ, Scott L. Electrocardiogram and rhythm strip interpretation by final year medical students. Ulster Med J. 2001;70(2):108-10.

12. Berger JS, Eisen L, Nozad V, D'Angelo J, Calderon Y, Brown DL, et al. Competency in electrocardiogram interpretation among internal medicine and emergency medicine residents. Am J Med. 2005;118(8):873-80.

13. de Jager J, Wallis L, Maritz D. ECG interpretation skills of South African Emergency Medicine residents. International journal of emergency medicine. 2010;3(4):309-14.

14. Eslava D, Dhillon S, Berger J, Homel P, Bergmann S. Interpretation of electrocardiograms by first-year residents: the need for change. Journal of electrocardiology. 2009;42(6):693-7.

15. Hoyle RJ, Walker KJ, Thomson G, Bailey M. Accuracy of electrocardiogram interpretation improves with emergency medicine training. Emerg Med Australas. 2007;19(2):143-50.

16. Margolis S, Reed R. EKG analysis skills of family practice residents in the United Arab Emirates: a comparison with US data. Fam Med. 2001;33(6):447-52.

17. Novotny T, Bond RR, Andrsova I, Koc L, Sisakova M, Finlay DD, et al. Data analysis of diagnostic accuracies in 12lead electrocardiogram interpretation by junior medical fellows. Journal of electrocardiology. 2015;48(6):988-94.

18. Pines JM, Perina DG, Brady WJ. Electrocardiogram interpretation training and competency assessment in emergency medicine residency programs. Acad Emerg Med. 2004;11(9):982-4.

19. Sibbald M, Davies EG, Dorian P, Yu EH. Electrocardiographic interpretation skills of cardiology residents: are they competent? Can J Cardiol. 2014;30(12):1721-4.

20. Trzeciak S, Erickson T, Bunney EB, Sloan EP. Variation in patient management based on ECG interpretation by emergency medicine and internal medicine residents. The American journal of emergency medicine.

2002;20(3):188-95.

21. Goy JJ, Schlaepfer J, Stauffer JC. Competency in interpretation of 12-lead electrocardiogram among Swiss doctors. Swiss medical weekly. 2013;143:w13806.

22. Mabuza LH, Mntla PS. Generalist practitioners' self-rating and competence in electrocardiogram interpretation in South Africa. Afr J Prim Health Care Fam Med. 2020;12(1):e1-e7.

23. Mant J, Fitzmaurice DA, Hobbs FD, Jowett S, Murray ET, Holder R, et al. Accuracy of diagnosing atrial fibrillation on electrocardiogram by primary care practitioners and interpretative diagnostic software: analysis of data from screening for atrial fibrillation in the elderly (SAFE) trial. BMJ. 2007;335(7616):380.

24. Pope JH, Aufderheide TP, Ruthazer R, Woolard RH, Feldman JA, Beshansky JR, et al. Missed diagnoses of acute cardiac ischemia in the emergency department. N Engl J Med. 2000;342(16):1163-70. 
25. Veronese G, Germini F, Ingrassia S, Cutuli O, Donati V, Bonacchini L, et al. Emergency physician accuracy in interpreting electrocardiograms with potential ST-segment elevation myocardial infarction: Is it enough? Acute cardiac care. 2016;18(1):7-10.

26. Cook DA, Oh SY, Pusic MV. Accuracy of Physicians' Electrocardiogram Interpretations: A Systematic Review and Meta-analysis. JAMA Intern Med. 2020;180(11):1-11.

27. Viljoen CA, Millar RS, Manning K, Burch VC. Determining electrocardiography training priorities for medical students using a modified Delphi method. BMC Med Educ. 2020;20(431):1-17.

28. Jablonover RS, Stagnaro-Green A. ECG as an Entrustable Professional Activity: CDIM Survey Results, ECG Teaching and Assessment in the Third Year. Am J Med. 2016;129(2):226-30 e1.

29. Keller D, Zakowski L. An effective ECG curriculum for third-year medical students in a community-based clerkship. Med Teach. 2000;22(4):354-8.

30. Patocka C, Turner J, Wiseman J. What adult electrocardiogram (ECG) diagnoses and/or findings do residents in emergency medicine need to know? CJEM. 2015;17(6):601-8.

31. Antiperovitch P, Zareba W, Steinberg JS, Bacharova L, Tereshchenko LG, Farre J, et al. Proposed In-Training Electrocardiogram Interpretation Competencies for Undergraduate and Postgraduate Trainees. J Hosp Med. 2018;13(3):185-93.

32. Auseon AJ, Schaal SF, Kolibash AJ, Jr., Nagel R, Lucey CR, Lewis RP. Methods of teaching and evaluating electrocardiogram interpretation skills among cardiology fellowship programs in the United States. Journal of electrocardiology. 2009;42(4):339-44.

33. Balady GJ, Bufalino VJ, Gulati M, Kuvin JT, Mendes LA, Schuller JL. COCATS 4 Task Force 3: Training in Electrocardiography, Ambulatory Electrocardiography, and Exercise Testing. J Am Coll Cardiol. 2015;65(17):176377.

34. Myerburg RJ, Chaitman BR, Ewy GA, Lauer MS. Task force 2: training in electrocardiography, ambulatory electrocardiography, and exercise testing. J Am Coll Cardiol. 2008;51(3):348-54.

35. Salerno SM, Alguire PC, Waxman HS, American College of P. Training and competency evaluation for interpretation of 12-lead electrocardiograms: recommendations from the American College of Physicians. Annals of internal medicine. 2003;138(9):747-50.

36. Wood G, Batt J, Appelboam A, Harris A, Wilson MR. Exploring the impact of expertise, clinical history, and visual search on electrocardiogram interpretation. Med Decis Making. 2014;34(1):75-83.

37. Breen C, Bond R, Finlay D. A clinical decision support tool to assist with the interpretation of the 12-lead electrocardiogram. Health Informatics J. 2019;25(1):51-61.

38. Norman GR, Brooks LR, Coblentz CL, Babcook CJ. The correlation of feature identification and category judgments in diagnostic radiology. Memory \& cognition. 1992;20(4):344-55.

39. Allen SW, Norman GR, Brooks LR. Experimental studies of learning dermatologic diagnosis: the impact of examples. Teaching and Learning in Medicine: An International Journal. 1992;4(1):35-44.

40. Grum CM, Gruppen LD, Woolliscroft JO. The influence of vignettes on EKG interpretation by third-year students. Acad Med. 1993;68(10 Suppl):S61-3.

41. Hatala R, Norman GR, Brooks LR. Impact of a clinical scenario on accuracy of electrocardiogram interpretation. Journal of general internal medicine. 1999;14(2):126-9.

42. Hatala RA, Norman GR, Brooks LR. The effect of clinical history on physicians' ECG interpretation skills. Acad Med. 1996;71(10 Suppl):S68-70. 
43. Sibbald M, Panisko D, Cavalcanti RB. Role of clinical context in residents' physical examination diagnostic accuracy. Med Educ. 2011;45(4):415-21.

44. Yardley S, Teunissen PW, Dornan T. Experiential learning: transforming theory into practice. Med Teach. 2012;34(2):161-4.

45. Maudsley G, Strivens J. Promoting professional knowledge, experiential learning and critical thinking for medical students. Med Educ. 2000;34(7):535-44.

46. Kennedy S, Simon B, Alter HJ, Cheung P. Ability of physicians to diagnose congestive heart failure based on chest X-ray. The Journal of emergency medicine. 2011;40(1):47-52.

47. Wood G, Knapp KM, Rock B, Cousens C, Roobottom C, Wilson MR. Visual expertise in detecting and diagnosing skeletal fractures. Skeletal Radiol. 2013;42(2):165-72.

48. Hatala R, Norman GR, Brooks LR. Influence of a Single Example on Subsequent Electrocardiogram Interpretation. Teaching and Learning in Medicine. 1999;11(2):110-7.

49. Hatala R, Gutman J, Lineberry M, Triola M, Pusic M. How well is each learner learning? Validity investigation of a learning curve-based assessment approach for ECG interpretation. Adv Health Sci Educ Theory Pract. 2019;24(1):45-63.

50. Hatala RM, Brooks LR, Norman GR. Practice makes perfect: the critical role of mixed practice in the acquisition of ECG interpretation skills. Adv Health Sci Educ Theory Pract. 2003;8(1):17-26.

51. Schumacher DJ, Englander R, Carraccio C. Developing the master learner: applying learning theory to the learner, the teacher, and the learning environment. Acad Med. 2013;88(11):1635-45.

52. Ramos D, Grad R, Saroyan A, Nugus P. Seeking coherence between 'mobile learning' applications and the everyday lives of medical residents. Perspectives on medical education. 2019;8(3):152-9.

53. Traxler J. Defining, Discussing and Evaluating Mobile Learning: The moving finger writes and having writ. The International Review of Research in Open and Distributed Learning. 2007;8(2).

54. Walsh K. Mobile Learning in Medical Education: Review. Ethiopian journal of health sciences. 2015;25(4):363-6.

55. Viljoen CA, Millar RS, Hoevelmann J, Muller E, Hähnle L, Manning K, et al. Utility of mobile learning in Electrocardiography. European Heart Journal-Digital Health. 2021.

56. Viljoen CA, Scott Millar R, Engel ME, Shelton M, Burch V. Is computer-assisted instruction more effective than other educational methods in achieving ECG competence amongst medical students and residents? A systematic review and meta-analysis. BMJ Open. 2019;9(11):e028800.

57. Viljoen CA, Millar RS, Manning K, Burch VC. Effectiveness of blended learning versus lectures alone on ECG analysis and interpretation by medical students. BMC Med Educ. 2020;20(1):1-16.

58. Bleakley A, Brennan N. Does undergraduate curriculum design make a difference to readiness to practice as a junior doctor? Med Teach. 2011;33(6):459-67.

59. Viljoen CA, Scott Millar R, Engel ME, Shelton M, Burch V. Is computer-assisted instruction more effective than other educational methods in achieving ECG competence among medical students and residents? Protocol for a systematic review and meta-analysis. BMJ Open. 2017;7(12):e018811.

60. Hurst JW. Methods used to interpret the 12-lead electrocardiogram: Pattern memorization versus the use of vector concepts. Clin Cardiol. 2000;23(1):4-13.

61. Burke JF, Gnall E, Umrudden Z, Kyaw M, Schick PK. Critical analysis of a computer-assisted tutorial on ECG interpretation and its ability to determine competency. Med Teach. 2008;30(2):e41-8. 
62. Kopeć G, Magoń W, Hołda M, Podolec P. Competency in ECG Interpretation Among Medical Students. Med Sci Monit. 2015;21:3386-94.

Figures

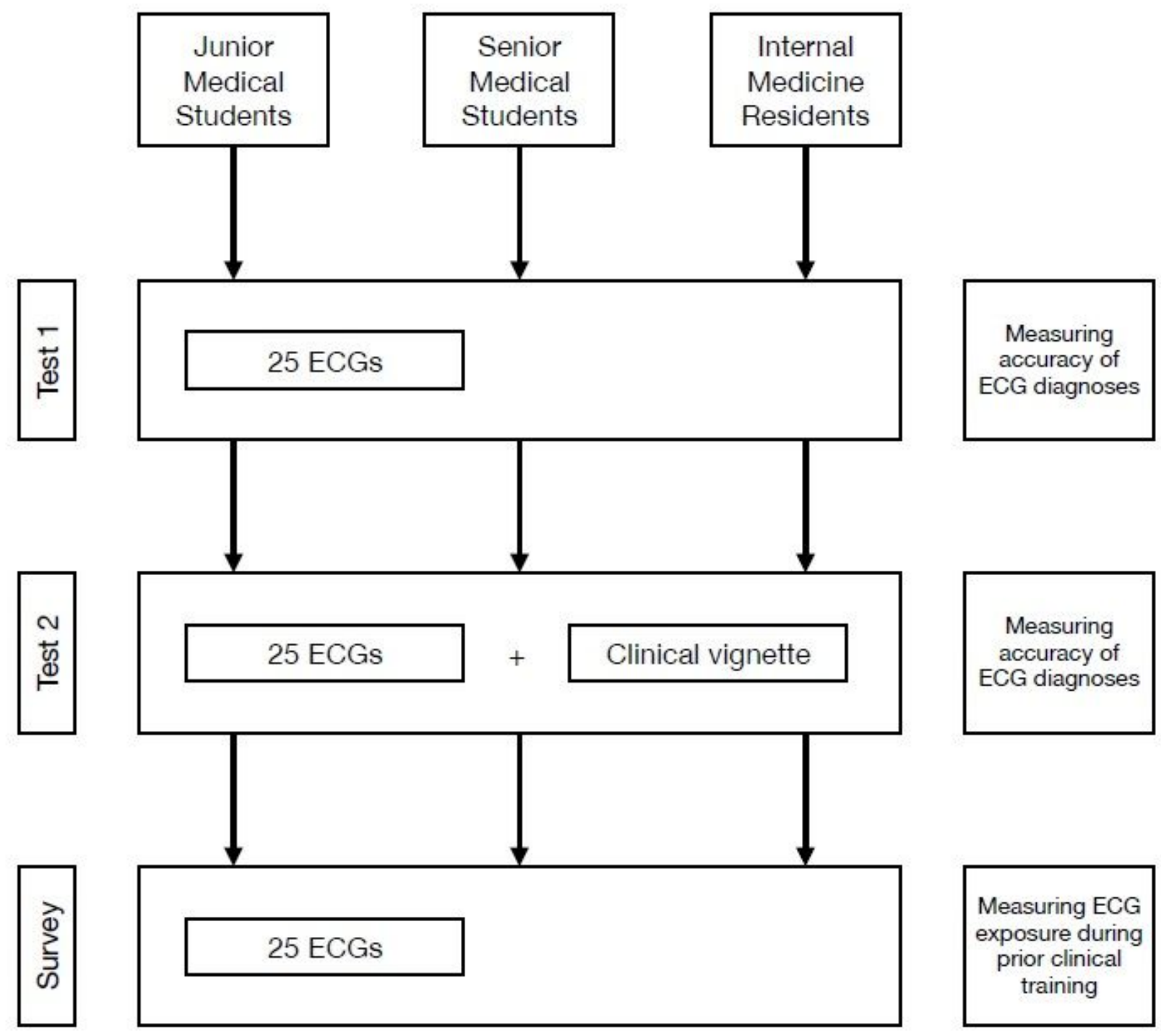

Figure 1

Study flow. The 25 ECGs included in the two tests and survey were 12 rhythm abnormalities (sinus arrhythmia, sinus arrest with escape rhythm, first degree AV block, Mobitz I and II second degree AV block, third degree AV block, atrial fibrillation [AF] with normal and uncontrolled rate, atrial flutter, AV node re-entrant tachycardia [AVNRT], ventricular tachycardia [VT] and ventricular fibrillation [VF]), and 13 waveform abnormalities (left and right atrial enlargement, left ventricular hypertrophy [LVH], right ventricular hypertrophy [RVH], left bundle branch block [LBBB], right bundle branch block [RBBB], left anterior fascicular block, Wolff-Parkinson-White [WPW] pattern, anterior and inferior STsegment elevation myocardial infarction [STEMI], pericarditis, hyperkalaemia, long QT syndrome. 
A

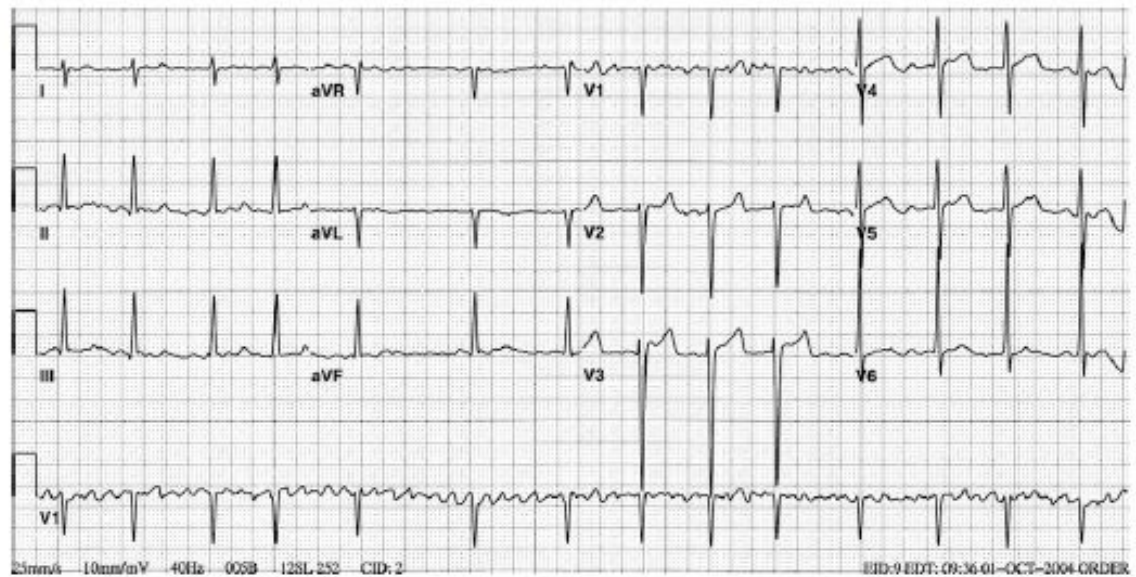

\begin{tabular}{|l|l|}
\hline \multicolumn{2}{|l|}{ An ECG is done on a 68 -year-old man with hypertension who presents with a stroke. What is your diagnosis? } \\
\hline x & a. Atrial fibrillation \\
\hline & b. Atrial flutter with variable AV block \\
\hline & c. Mobitz type I AV block \\
\hline & d. Mobitz type II AV block \\
\hline & e. I do not know the answer \\
\hline
\end{tabular}

B

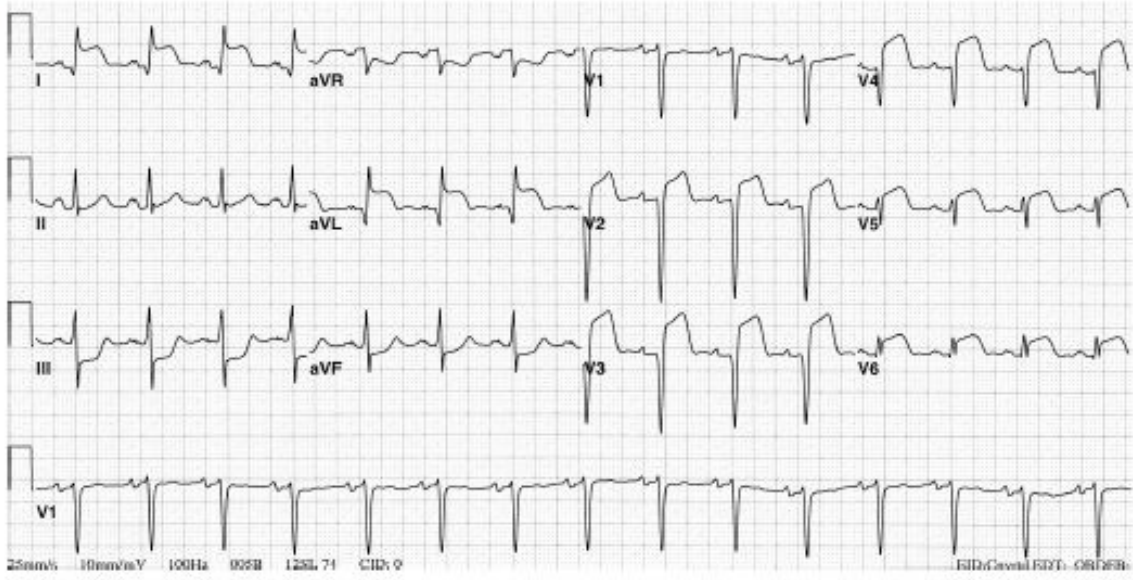

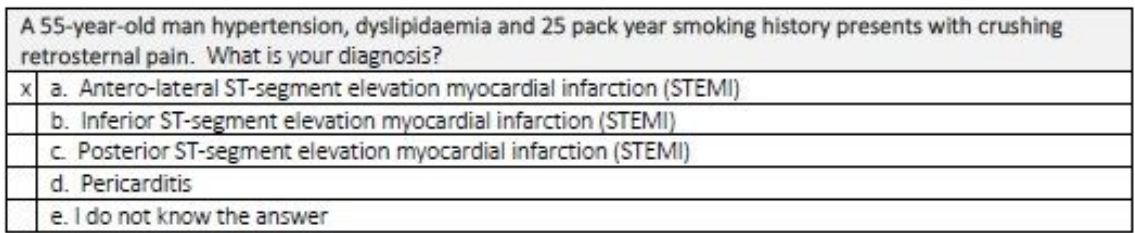

\section{Figure 2}

An example of the ECG, case vignette and multiple-choice questions asked for (A) rhythm abnormalities and (B) waveform abnormalities. Case vignettes were only shown in Test 2. 


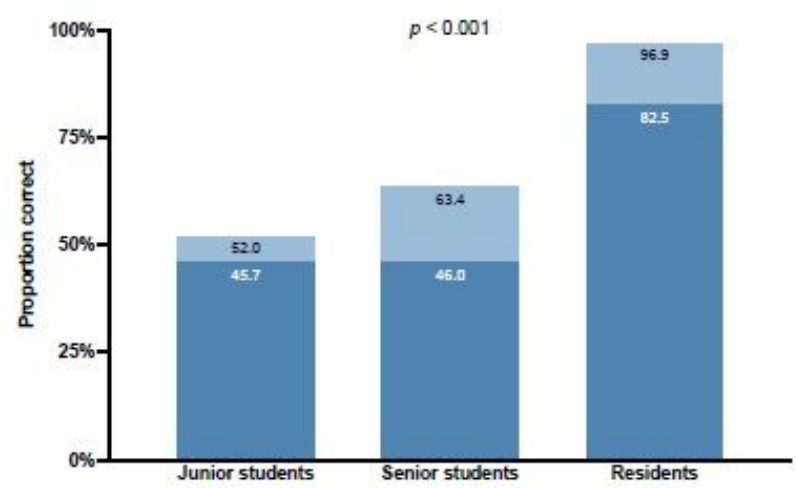

B Arrhythmias
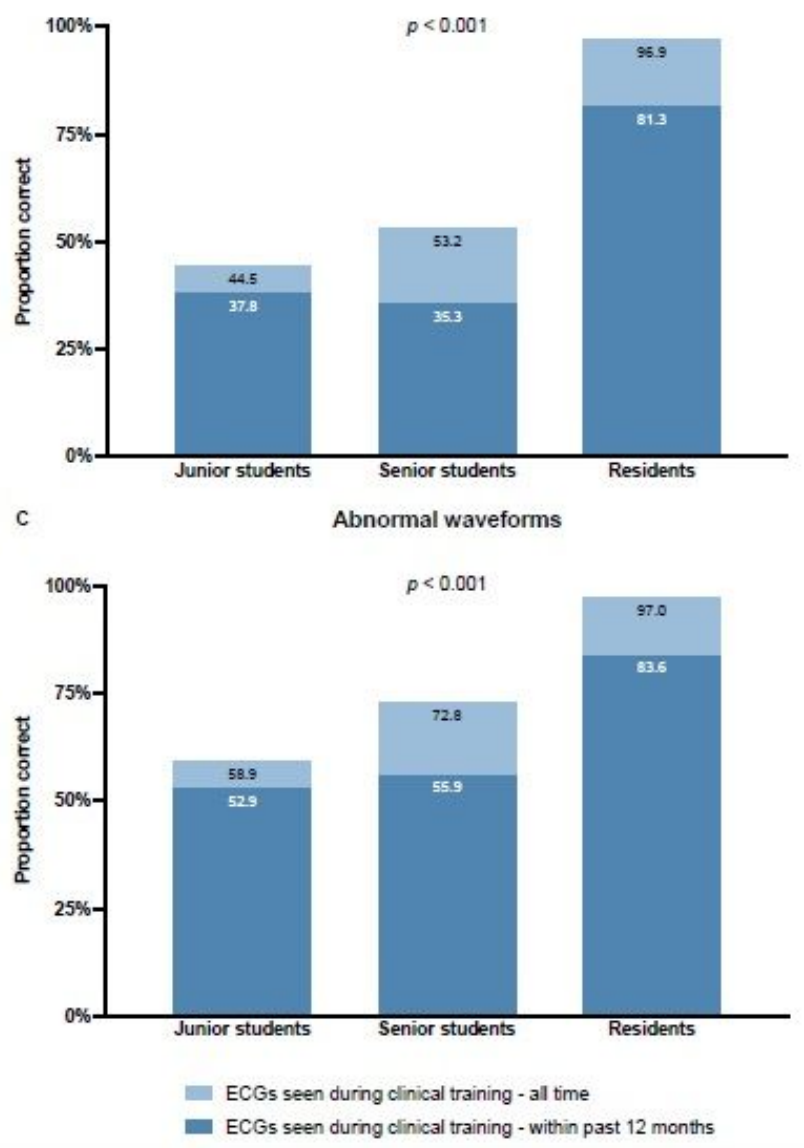

Figure 3

Exposure to ECGs during clinical training, for (A) all ECGs tested, (B) arrhythmias and (C) waveform abnormalities. 


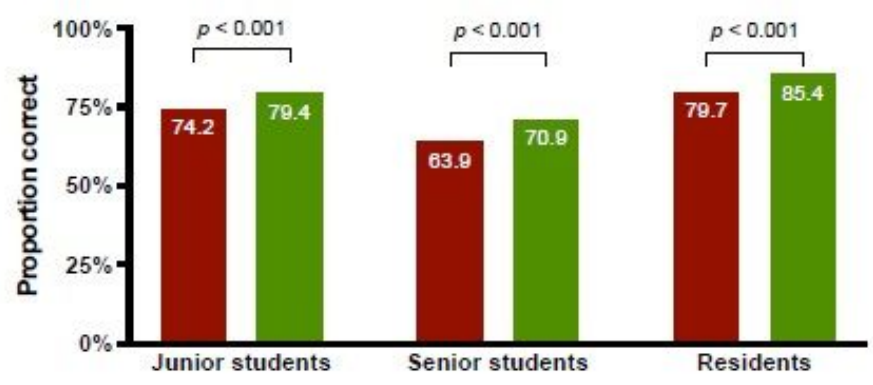

B

Arrhythmias

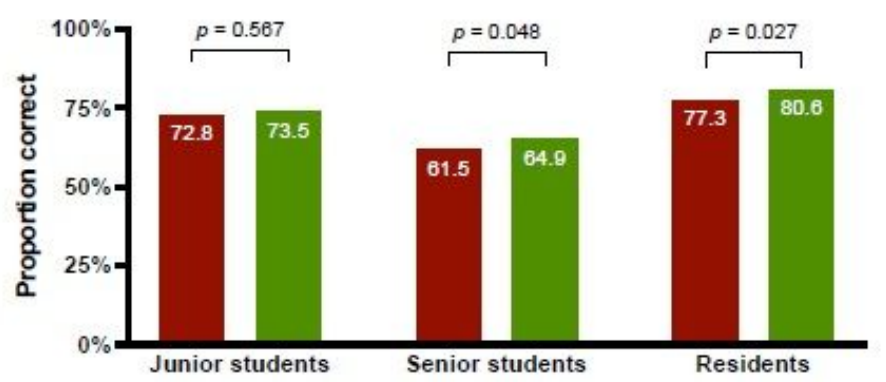

C Abnormal waveforms

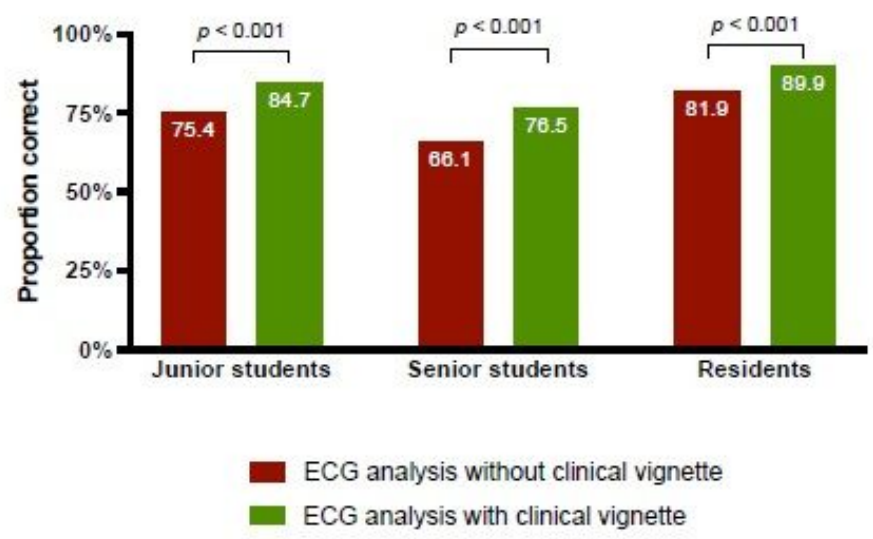

Figure 4

Difference of ECG diagnostic accuracy when participants were provided with a case vignette or not, for (A) all ECGs tested, (B) arrhythmias and (C) waveform abnormalities. 


\section{ECG interpretation without a clinical vignette}

Junior students

$1.44(1.16-1.79)$

Senior students

Medical residents

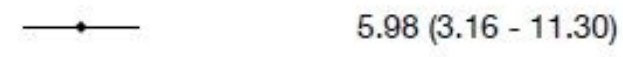

Overall $\quad\left(R^{2}=88.8 \%\right)$

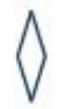

$1.47(1.17-1.84)$

$5.98(3.16-11.30)$

$1.58(1.35-1.84)$

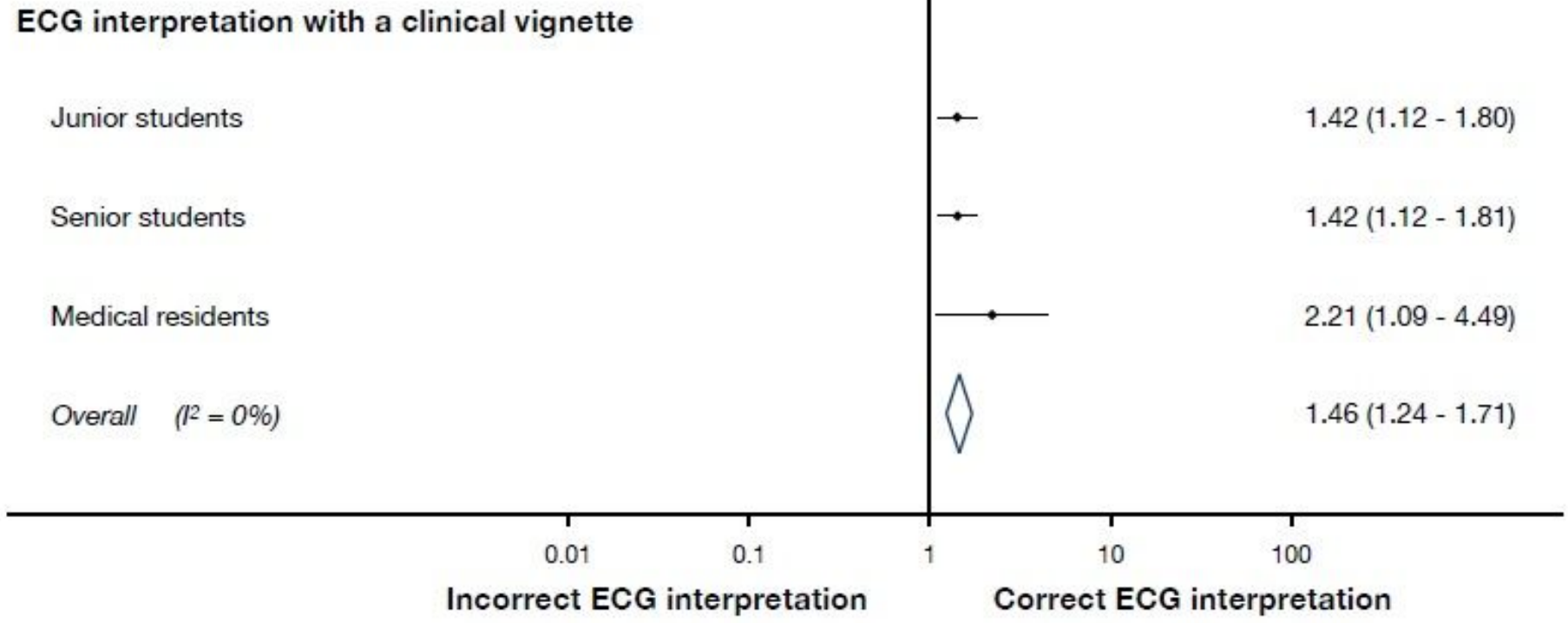

Figure 5

Association between prior clinical exposure and ECG diagnostic accuracy, when participants were provided with a case vignette or not.

\section{Supplementary Files}

This is a list of supplementary files associated with this preprint. Click to download.

- supplementarymaterialECGtest.pdf 